\title{
Comparison of a Physiotherapy Program versus Dexamethasone Injections for Plantar Fasciopathy in Prolonged Standing Workers: A Randomized Clinical Trial
}

Running Head: Physio versus Steroid RCT in Workers

Michael Ryan PhD ${ }^{1,2, *}$, Jamie Hartwell BKin ${ }^{1}$, Scott Fraser $\mathrm{PT}^{1}$, Richard Newsham-West MPhty, PhD ${ }^{2}$, Jack Taunton MD ${ }^{1}$

${ }^{1}$ Allan McGavin Sports Medicine Centre, University of British Columbia, Vancouver, Canada

${ }^{2}$ Griffith Health Institute, Griffith University, Southport, Australia

* Corresponding Author: Clinical Sciences 1, Gold Coast Campus, Griffith University, Southport, Australia 4222

Phone: +61 755527443

Fax: +61 755528674

Email: mryan76@gmail.com

Funding: Innovation at Work Grant, WorkSafeBC, \# RS2010-IG20 ClinicalTrials.gov Identifier: NCT01297686

Word Count: 2997

Abstract Word Count: 250

Keywords: Plantar fascia, plantar fasciitis, physiotherapy, steroid injection, worker 


\section{Acknowledgements}

The research team would like to thank the support of The BC Nurses Union, BC Federation of Labour, the BC Teacher's Federation, London Drugs and Vancouver Coastal Health Authority for their assistance in spreading the word about this study to their members. We also would like to thank our volunteers for their valuable help in data collection: Jason Wong, Hansel Yu, Jeremy Jung, Amanda Mow and Maha Elashi. This research is supported with funds from WorkSafeBC through the Focus on Tomorrow program. 


\begin{abstract}
Objective: To investigate the effectiveness of a physiotherapy-based exercise program versus dexamethasone injection for chronic plantar fasciopathy in workers standing for prolonged periods of time.
\end{abstract}

Design: A parallel group non-blinded randomized controlled trial with 12-week follow-up.

Setting: An outpatient sports medicine clinic in Vancouver, Canada.

Participants: 56 workers required to stand for greater than 5 hours per day with chronic plantar fasciopathy took part. Diagnosis from a physiotherapist must include signs of structural changes to the plantar fascia seen on ultrasound.

Interventions: The PHYSIO group included 7 physiotherapy-lead exercises performed daily over a 12-week period. The INJECTION group received one palpation-guided dexamethasone injection, followed by a daily routine of calf stretching.

Main Outcome Measures: The Foot and Ankle Disability Index (FADI) scores 12 weeks post-intervention and ultrasound based measures of ligament appearance.

Results: At follow-up both groups reported significant improvements in FADI and visual analog scales for pain at work and with activities of daily living at 6 and 12 weeks compared to baseline scores $(p<0.001)$. There were no significant between group differences. There were no significant changes to plantar fascia thickness reported at the 6 and 12 week follow-up point. Both the number of cases with focal anechoic areas and the size of these anechoic areas improved significantly in the PHYSIO $(p=0.003)$ and INJECTION $(p<0.001)$ groups at 12-week follow-up. 
Conclusion: Workers standing for prolonged periods experienced the same shortterm therapeutic effectiveness with a physiotherapy lead exercise program compared to an injection of corticosteroid with stretching. 
Chronic plantar fasciopathy is a painful, degenerative condition typically with a focal point of tenderness at the medial plantar heel and medial longitudinal arch of the foot. It is also a very common condition. Approximately 1 million patient visits per year were made to physicians in the 6-year period from 1995-2000 for the diagnosis and treatment of plantar fasciopathy.[1]

Plantar fasciopathy is common amongst individuals in the workplace with job descriptions requiring prolonged standing highly represented.[2] In a study of over 500 supermarket workers, the highest incidence of foot injuries occurred in workers who had the highest frequency of standing at their job, and individuals who report standing for the majority of the day are over $300 \%$ more likely to develop heel pain than those who did not.[3, 4] Similar findings are reported in the automobile industry.[5]

The cause of plantar fasciopathy is both multifactorial and dependent on individual history. The specific role that prolonged standing has on the onset of injury is speculative; however, it is reasonable that tissue overload plays a role. The plantar fascia ligament is a thick band of dense connective tissue that provides support for the longitudinal arch of the foot during weight-bearing by forming a truss spanning the calcaneus through the metatarsophalangeal joints with divisions attaching at the distal phalanges. This broad thick ligament therefore undergoes tensile loading when body weight is applied to the foot. Prolonged standing fatigues lower limb musculature that is shown to affect postural control and muscle activation patterns that in turn may inappropriately load plantar soft tissue.[6] An additional component may be fatigue of plantar intrinsic muscles such a flexor Hallucis brevis $m$. and abductor Hallucis $m$. which are known to provide dynamic support for the medial 
longitudinal arch, although this association has not been reported specific to a prolonged standing task.[7]

Treatment options for workers with plantar fasciopathy are varied and there is little consensus on clinical approach, especially when rest is not an option. Steroid injections are a common treatment for plantar fasciopathy stemming from positive evidence they are effective at short-term management of symptoms.[8-11] By their nature corticosteroids are not regenerative, are directed primarily at producing relief of pain, and have limited evidence supporting long-term treatment effectiveness.[8,

12] Few other therapies have been as extensively researched. Surprisingly, exercise has only received sparse attention in the medical literature despite the fact, in one form or another, it is arguably the most common treatment approach used to address chronic heel pain. Furthermore, exercise-based treatment options for plantar fasciopathy offer promise in that their focus is on restoring tissue function, rather than simply symptom reduction. Histological reports from surgical biopsies of the plantar fascia do not demonstrate inflammatory infiltrates, but rather mycoid or fibrocartilaginous tissue that is representative of an aberrant remodeling process.[13] Accordingly, treatment efforts aimed at restoring ligament function and improving lower extremity coordination (to better distribute stress away from the plantar fascia) should be a priority. Both steroid injections and exercise are common therapies yet no study has directly compared these two interventions in the same study.

\section{Methods}

We carried out a parallel group, randomized controlled trial to investigate the effectiveness of a physiotherapy-directed multi-element exercise program versus a 
steroid injection and calf stretch treatment at reducing the symptoms of chronic plantar fasciopathy in workers who must stand for prolonged periods of time. All procedures within this study were approved by the Clinical Research Ethics Board at the University of British Columbia.

\section{Setting and eligibility criteria}

The current study was carried out at the University of British Columbia (Vancouver, Canada) between May 2011 and March 2012. Recruitment was performed through various sources, including newspaper advertisements, distribution through respective workplaces, and word-of-mouth. Participants were required to have a history of inferior heel pain for at least 12 months and to report a minimum heel pain of $20 \mathrm{~mm}$ on a $100 \mathrm{~mm}$ visual analog scale and have pain through direct palpation of the medial calcaneal tubercle or proximal plantar fascia.

Diagnostic ultrasound confirmed the diagnosis of plantar fasciopathy.[14] During the ultrasound exam the plantar fascia and surrounding tissue was examined (Logiq-e, GE) in the longitudinal and transverse planes using a $12-5 \mathrm{MHz}$ linear array probe.

We excluded potential recruits if they had received a corticosteroid injection for plantar heel pain within the previous six months, had a known hypersensitivity to lidocaine hydrochloride or corticosteroids, current skin or soft tissue infection near the possible injection site, inflammatory disease, diabetes mellitus, previous local surgery, a history of local trauma or other musculoskeletal condition that might impair function of the foot or ankle. We excluded individuals who are involved in litigation for their heel pain or on worker's compensation benefits.

\section{Interventions}




\section{Physiotherapy Group}

The physiotherapy program (PHYSIO) included one consultation with a therapist (author SF) at the beginning of the study to review workplace conditions, discuss relevant clinical history and overview the exercise program.

Detailed instruction for each exercise was given and participants needed to have demonstrated mastery (confirmed by physiotherapist SF) of at least the preliminary technique prior to involvement in the study. Mastery was considered achieved when participants demonstrated smooth, controlled motions that progressed through the appropriate range for that exercise.

The exercise regimen in the PHYSIO group included the following 7 exercises performed on both right and left sides daily over a 12-week period (Figure 1):

- Karaoke: lateral side step movement involving crossing one foot over the next for 5 sets of 15 cross-overs in each direction;

- Balance walking, or walking along a straight line on the ground, for 5 sets of 30 strides;

- Forefoot extension exercise: participant stands feet shoulder width apart with one foot ahead of the other and then contracting only calf muscles of the back leg, lifts the heel of the back leg until the metatarsophalangeal joint of that foot is maximally extended for 5 sets of 15 repetitions.

- Standing one-legged balance exercise: performed initially with eyes open, then with eyes closed on the ground, then on an unstable surface for 1 minute. 
- Ankle inversion/eversion exercise: foot is placed sideways at the edge of a step. After stabilizing the remainder of the foot and leg, the ankle is inverted and everted to the limits of the range for 3 sets of 15 repetitions.

- Gastrocnemius and soleus stretching: while standing in a neutral position and the knee extended the foot is placed on top of a ramp elevating the forefoot on the rearfoot (talocrural dorsiflexion) and held for 3 sets of 30 seconds each. Next the foot is again placed on top of a phone-book with the knee flexed approximately $15-20$ degrees and held for 3 sets of 30 seconds each.

- Tissue-specific plantar fascia stretch: in a sitting position the right foot is crossed over the left while one hand passively extends the right forefoot. The left hand then applies light to moderate pressure in 3-5 second intervals along the length of the medial longitudinal arch.

Compliance with the physiotherapy regimen was confirmed with an on-line training log participants submitted on a weekly basis.

\section{Injection Group}

The INJECTION group received a palpation-guided corticosteroid injection by a sports-medicine fellowship trained physician (JT) with over 30 years' clinical experience. The steroid injection procedure has been described previously in the literature.[10] A 22-guage, 1.5" needle and $3 \mathrm{~cm}^{3}$ syringe filled with $1 \mathrm{ml}$ of dexamethasone mixed with $0.5 \mathrm{ml}$ of $1 \%$ lidocaine was prepared. Prior to injection, the skin was sterilized with povidone-iodine. The needle was inserted $2-3 \mathrm{~cm}$ anteromedial to the focal point of pain in the inferior heel near the calcaneal tuberosity and moved toward the most tender area. Both feet of participants with bilateral plantar fasciopathy were injected during one appointment. We advised 
participants to avoid running and other high impact activities for the two weeks following the injection.

Participants in the injection group were also asked to complete a daily calf stretching programme (see gastrocnemius/soleus stretch exercise description above) to ensure that the trial better represented normal clinical practice. Compliance with stretches was recorded on an on-line training log completed weekly.

\section{Randomization and treatment allocation}

Treatment allocation was performed using a computer generated block (block size 4) random number sequence. The investigator $(\mathrm{MR})$ who generated the random number sequence had no contact with participants throughout the trial. Allocation was not concealed to the investigator $(\mathrm{JH})$ collecting participant outcome measures.

\section{Outcome measures}

The primary clinical outcome measures used in this study include the Foot and Ankle Disability Index (FADI).[15] The clinimetric properties of the FADI have been reported previously.[16, 17]

Secondary clinical measures include measures of pain and sonography of the plantar fascia. Visual analog scale (VAS) items for worst pain experienced in the heel/arch area over the past 7 days while at work, and during activities of daily living (ADL) were reported.[18] The following sonographic outcomes were included: plantar fascial thickness, the presence and size of focal anechoic areas within the plantar fascia will be described below (Figure 2). Fascial thickness was recorded as the greatest distance spanning the fascia in the anterior-posterior plane. Intrafascial anechoic regions were measured in the longitudinal plane. 
All clinical and sonographic outcome measures were assessed at baseline and at 6 and 12 week follow-up. Treatment compliance and use of analgesic medication were recorded via questionnaire at the 6 and 12 week follow-up points.

\section{Data Analysis}

Our a-priori power analysis was based on preliminary data conducted using the same multi-element exercise protocol on a population with plantar fasciopathy, that reported an average baseline pain score of 52.8 with a standard deviation of 24.6.[19] The minimum clinically significant difference (MCID) has not been reported for the FADI; therefore, a $30 \%$ change in pain scores between groups is (difference in VAS scores of 15.84) will be considered a clinically significant difference. Based on these a-priori data, 39 participants were needed in each group with a 95\% probability of avoiding a type I error and a power level of 0.80 .

Descriptive statistics were carried out for initial group characterization and it was determined following independent samples t-tests that participant age and weight differed significantly across groups (table 1). A repeated measures analysis of variance through a general linear model was conducted to determine the withinsubject effect of time and between subject effect of group allocation, adjusting for participant age and weight in the model. A Fischer's least significant difference posthoc test differentiated significance across levels for each effect. Independent samples t-test compared between-group differences at specific time points. Chisquare tests were used to determine significant differences in each group across time in the categorical variable 'presence of anechoic areas'. The alpha for this study will be set at 0.05 for significance, with a near-significance threshold at $p$ values less than 0.10 . 


\section{Results}

From May 2011 to February 2012, 208 individuals were assessed for eligibility (Figure 3). Sixty-five individuals were enrolled in the study and underwent initial baseline testing and randomization, but from this sample 9 participants did not return for any follow-up testing despite repeated efforts from study personnel and are considered drop-outs for the purpose of analysis. Five participants (1 injection, 4 physiotherapy) were lost to follow-up (unable to contact after 5 attempts) after the 6 week follow-up point; data from these participants was included in the final analysis after applying an intention-to-treat approach. Missing data from these five participants was imputed using a last value carried forward strategy.[20]

In total, 56 patients with plantar fasciopathy were included in the final analysis (Table 1). Twelve participants in both groups presented with bilateral symptoms; in these cases the more severe side (based on VAS work scores) was reported. There were no significant differences in any of the clinical or sonographic measures at baseline across groups.

At follow-up both groups reported significant improvements in VAS Work, VAS ADL, and FADI outcomes at 6 and 12 weeks compared to their baseline scores (table 2).

There were no significant changes to plantar fascia thickness reported at the 6 and 12 week follow-up point (table 3). Both the number of cases with focal anechoic areas and the size of these anechoic areas improved significantly in both treatment groups at the 12-week follow-up.

\section{Discussion}


The objective of this study was to determine whether there was similar or superior effectiveness of a practical and work-place compatible exercise regimen for the treatment of plantar fasciopathy compared to a standard-of-care protocol of calf stretching following an injection of a corticosteroid. We report a clinical improvement that surpasses the minimum clinically important difference (MCID) for the VAS pain scales used in this study.[21] Positive structural improvements were also seen through ultrasound assessment from baseline to the 12-week follow-up in both treatment groups. Results from this study therefore suggest that performing a multielement exercise program may be equally as effective as receiving a steroid injection for addressing symptoms of plantar fasciopathy in the short term.

The mechanism behind therapeutic success with stretching exercises remains unknown with no clear answer as to the relationship between exercise intensity, speed, or frequency and clinical outcome[22]. We speculate that the clinical success experienced by the group performing the physiotherapy regimen in the present study is a result of 1 or a combination of the following 3 therapeutic effects. Firstly, the tissue-specific stretching and forefoot extension exercises provide direct and systematic loading to the plantar fascia. Appropriate loading of soft tissue is an important element for mechanotransduction related maintenance of extracellular matrix tensile strength.[23] The second effect comes from improved activation and strengthening of extrinsic foot muscles through the inversion/eversion, balance walking, and karaoke side-stepping exercises. Extrinsic foot musculature provides important dynamic support of the longitudinal arch, and direct activation of these extrinsic muscle groups could decrease stress on passive structures such as the plantar fascia.[7] A previous case report on a directed program of targeted exercises to increase range of motion and progressively increase sport-specific stress on the 
plantar fascia reported favourable clinical outcomes.[24] Lastly, two exercises were aimed at addressing overall posture and balance to minimize postural contributions to loading of the plantar fascia and increased calf tension (secondary to forward sway). Similar balance exercises incorporating semi-compressible foam rollers have shown to significantly improve dynamic balance.[25]

Previous authors have used components of the multi-element exercise program in this study and reported favourable findings. The tissue-specific stretching exercise first reported by DiGiovanni et al.(2003, 2006) showed a strong clinical improvement after both short and long term follow-up.[26, 27] Our own group showed a positive clinical improvement following use of the same multi-element protocol in a group using minimalist footwear; however, only a portion of that sample were workers exposed to the constant stress of standing for prolonged periods of time.[19]

Other groups have reported on the short and medium term effectiveness of corticosteroid injections (without ultrasound guidance) at reducing pain in a population with chronic heel pain.[11, 28, 29] Both Genc et al. (2005) and Kane et al. (2001) found a decrease in fascial thickness post-steroid (methylprednisone and triamcinolone hexacetonide, respectively) injection, but do not report changes to any other sonographic feature.[29, 30] The present study found no change in fascial thickness but substantial changes to the prevalence and size of anechoic regions at 12-week follow-up owing possibly to a different injectate (dexamethasone) and different sample demographics and tissue response following prolonged standing.

\section{Study Limitations}

Results from this study should be interpreted in light of some limitations. Most notably, there is no true control group. Blinding of the study personnel to treatment 
allocation was not performed making our outcomes vulnerable to bias.[20, 31] There is large variation in the symptom duration reported in both groups in this study suggesting that there may be different levels of pathology within this sample.[32]

The findings from this study have significant implications for worker health-related quality of life. The positive treatment effect in the physio group from the standpoint of restoring both pain and disability, but also plantar fascial structure, suggests workers may have a pragmatic option for achieving clinically significant improvements from plantar fasciopathy. The exercises used in this study are relatively simple to perform, are without side-effects or adverse events, and use equipment that, for the most part, is easy to find in the workplace. Furthermore, the short term clinical effectiveness of this multi-element protocol are equivalent to corticosteroid injection therapy, the only therapy supported through evidence from the Cochrane Group[33], but without exposing the worker to a steroid injection procedure and the associated risk of ligament rupture.[34, 35]

\section{References}

1. Riddle DL, Schappert SM. Volume of ambulatory care visits and patterns of care for patients diagnosed with plantar fasciitis: a national study of medical doctors. Foot Ankle Int. 2004; 25:303-10.

2. Taunton J, Ryan M, Clement D, et al. Plantar Fasciitis: a retrospective analysis of 267 cases. Physical Therapy in Sport. 2002; 3:57-65.

3. Riddle $D$, Pulisic $M$, Pidcoe $P$, et al. Risk factors for Plantar fasciitis: a matched case-control study. J Bone Joint Surg Am. 2003; 85-A:872-877.

4. Ryan G. The prevalence of musculo-skeletal symptoms in supermarket workers. Ergonomics. 1989; 32:359-371.

5. Werner R, Gell N, Hartigan A, et al. Risk factors for plantar fasciitis among assembly plant workers. PM\&R. 2010; 2:110-116.

6. Madeleine $P$, Voigt M, Arendt-Nielsen L. Subjective, physiological and biomechanical responses to prolonged manual work performed standing on hard and soft surfaces. Eur J Appl Physiol Occup Physiol. 1998; 77:1-9.

7. Headlee DL, Leonard JL, Hart JM, et al. Fatigue of the plantar intrinsic foot muscles increases navicular drop. J Electromyogr Kinesiol. 2008; 18:420-425. 
8. Ball EM, McKeeman HM, Patterson C, et al. Steroid injection for inferior heel pain: a randomised controlled trial. Ann Rheum Dis. 2012; 72:996-1002.

9. Crawford F, Atkins D, Young $P$, et al. Steroid injection for the treatment of plantar fasciitis: evidence of short term effectiveness. A randomised controlled trial. Rheumatology. 1999; 38:974-977.

10. Tsai WC, Hsu CC, Chen CP, et al. Plantar fasciitis treated with local steroid injection: comparison between sonographic and palpation guidance. J Clin Ultrasound. 2006; 34:12-16.

11. Tsai WC, Wang CL, Tang FT, et al. Treatment of proximal plantar fasciitis with ultrasoundguided steroid injection. Arch Phys Med Rehabil. 2000; 81:1416-1421.

12. Crawford F, Thomson C. Interventions for treating plantar heel pain. Cochrane Database Syst Rev. 2003.

13. Lemont $\mathrm{H}$, Ammirati KM, Usen N. Plantar fasciitis: a degenerative process (fasciosis) without inflammation. J Am Podiatr Med Assoc. 2003; 93:234-237.

14. McMillan AM, Landorf KB, Barrett JT, et al. Diagnostic imaging for chronic plantar heel pain: a systematic review and meta-analysis. J Foot Ankle Res. 2009; 2:32.

15. Martin R, Burdett R, Irrgang J. Development of the Foot and Ankle Disability Index (FADI) [abstract]. J Orthop Sports Phys Ther. 1999; 29:A32-33.

16. Hale SA, Hertel J. Reliability and Sensitivity of the Foot and Ankle Disability Index in Subjects With Chronic Ankle Instability. J Athl Train. 2005; 40:35-40.

17. Eechaute $C$, Vaes $P$, Van Aerschot $L$, et al. The clinimetric qualities of patient-assessed instruments for measuring chronic ankle instability: a systematic review. BMC Musculoskelet Disord. 2007; 8:6.

18. Boonstra A, Schiphorst Preuper $H$, Reneman $M$, et al. Reliability and validity of the visual analogue scale for disability in patients with chronic musculoskeletal pain. Int J Rehabil Res. 2008; 31:165-169.

19. Ryan M, Fraser S, McDonald K, et al. Examining the degree of pain reduction using a multielement exercise model with a conventional training shoe versus an ultraflexible training shoe for treating plantar fasciitis. Phys Sportsmed. 2009; 37:68-74.

20. Friedman L, Furberg C, DeMets D. Fundamentals of Clinical Trials 3rd ed. New York, NY: Springer; 1998.

21. Farrar JT, Young JP, Jr., LaMoreaux L, et al. Clinical importance of changes in chronic pain intensity measured on an 11-point numerical pain rating scale. Pain. 2001; 94:149-158.

22. Rompe JD, Cacchio A, Weil L, Jr., et al. Plantar fascia-specific stretching versus radial shockwave therapy as initial treatment of plantar fasciopathy. J Bone Joint Surg Am. 2010; 92:2514-2522.

23. Wall ME, Banes AJ. Early responses to mechanical load in tendon: role for calcium signaling, gap junctions and intercellular communication. J Musculoskelet Neuronal Interact. 2005; 5:70-84.

24. Ross M. Use of the tissue stress model as a paradigm for developing an examination and management plan for a patient with plantar fasciitis. J Am Podiatr Med Assoc. 2002; 92:499506.

25. Bellew JW, Fenter PC, Chelette B, et al. Effects of a short-term dynamic balance training program in healthy older women. J Geriatr Phys Ther. 2005; 28:4-8, 27.

26. DiGiovanni B, Nawoczenski D, Lintal M, et al. Tissue-specific plantar fascia-stretching exercise enhances outcomes in patients with chronic heel pain. A prospective, randomized study. J Bone Joint Surg Am. 2003; 85-A:1270-1277.

27. DiGiovanni B, Nawoczenski D, Malay D, et al. Plantar fascia-specific stretching exercise improves outcomes in patients with chronic plantar fasciitis. A prospective clinical trial with two-year follow-up. J Bone Joint Surg Am. 2006; 88:1775-1781. 
28. Kalaci A, Cakici H, Hapa O, et al. Treatment of plantar fasciitis using four different local injection modalities: a randomized prospective clinical trial. J Am Podiatr Med Assoc. 2009; 99:108-113.

29. Genc H, Saracoglu M, Nacir B, et al. Long-term ultrasonographic follow-up of plantar fasciitis patients treated with steroid injection. Joint Bone Spine. 2005; 72:61-65.

30. Kane $\mathrm{D}$, Greaney $\mathrm{T}$, Shanahan $\mathrm{M}$, et al. The role of ultrasonography in the diagnosis and management of idiopathic plantar fasciitis. Rheumatology. 2001; 40:1002-1008.

31. Roethlisberger F, Dickson W. Management and the Worker: An Account of a Research Program Conducted by Western Electric Company, Hawthorne Works, Chicago. Cambridge, Massachusetts: Harvard University Press; 1939.

32. Dunn KM, Croft PR. The importance of symptom duration in determining prognosis. Pain. 2006; 121:126-32.

33. Hawke F, Burns J, Radford J, et al. Custom-made foot orthoses for the treatment of foot pain. Cochrane Database Syst Rev. 2008; 3.

34. Acevedo JI, Beskin JL. Complication of plantar fascia rupture associated with corticosteroid injection. Foot Ankle Int. 1998; 19:91-97.

35. Sellman JR. Plantar fascia rupture associated with corticosteroid injection. Foot Ankle Int. 1994; 15:376-381.

\section{Figure Captions}

Figure 1 - Composite illustration of the 7 exercise components included in the multielement treatment protocol: A) tissue specific plantar fascia stretch; B) balance walking; C) karaoke side-stepping; D) forefoot extension exercise; E) calf stretch with aid of sloped surface; F) single-legged balance exercise; G) ankle inversion/eversion exercise

Figure 2 - Ultrasound image of the plantar fascia (PF) seen at its insertion to the calcaneus (C) deep to plantar fat pad (FP). An anechoic region is seen distal to insertion (arrow)

Figure 3 - CONSORT flow diagram of participant recruitment and group allocation 